\title{
CORRELATION OF THE NUMBER OF IMAGES OF AN N-POINT GRAVITATIONAL LENS AND THE NUMBER OF SOLUTIONS OF ITS SYSTEM
}

\author{
A.T. Kotvytskiy ${ }^{1,2}$, S.D. Bronza ${ }^{2}$, V.Yu. Shablenko ${ }^{1}$ \\ ${ }^{1}$ Karazin Kharkov National University, \\ Svobody Square 4, Kharkiv, 61022, Ukraine, kotvytskiy@gmail.com \\ ${ }^{2}$ Ukrainian State University of Railway Transport, \\ Feierbakh Square 7, 61050, Kharkiv, Ukraine bronza_semen@mail.ua
}

\begin{abstract}
In this paper, we study the correlation between the number of solutions of a system of lens equations and the number of source images that a gravitational lens has. We defined the concept of an image in a gravitational lens. To find the set of solutions of a system of lens equations, we applied methods of algebraic geometry.
\end{abstract}

Keywords: Lens: gravitational lenses, images, solutions; Algebraic geometry: resultant, Bezout theorem.

\section{Introduction}

One of the main theoretical problems of gravitational lensing is the study of images. This problem is studied in some publications (Bliokh et al., 1989; Zakharov, 1997; Schneider, 1999). The images in these publications are studied mostly by numerical methods, sometimes with elements of an analytical description. In this paper, we study the images of a point source in N-point gravitational lens analytically. In this paper, we continue to investigate gravitational lenses by methods of algebraic geometry. The results obtained by these methods previously published in (Kotvytskiy \& Bronza, 2016; Kotvytsky et al., 2016; Kotvytskiy et al., 2017; Bronza \& Kotvytskiy, 2017).

\section{Images in gravitational lensing}

In astrophysical literature, the concept of an image in a gravitational lens is usually not defined. In terms of empirical astrophysics this concept is obvious. However, the absence of a definition can lead to ambiguous understanding of the concept and a different interpretation of some results, for example, the theorem on the odd number of images (Zakharov, 1997; Schneider, 1999). The terminology developed in algebraic geometry enables us to define the concept of an image in a gravitational lens. Based on this definition, we can refine certain known statements and formulate new ones.

The equation of N-point lens (in dimensionless form) has the form:

$$
\vec{y}=\vec{x}-\sum_{i} m_{i} \frac{\vec{x}-\vec{x}_{i}}{\left|\vec{x}-\vec{x}_{i}\right|^{2}},
$$

where $\vec{x}_{i}$ - are the radius vectors of the point masses entering the lens, and $m_{i}, \sum_{i} m_{i}=1-$ their dimensionless masses.

Equation (3) in the coordinate form has the form:

$$
\left\{\begin{array}{c}
y_{1}=x_{1}-\sum_{i=1}^{N} m_{i} \frac{x_{1}-a_{i}}{\left(x_{1}-a_{i}\right)^{2}+\left(x_{2}-b_{i}\right)^{2}} \\
y_{2}=x_{2}-\sum_{i=1}^{N} m_{i} \frac{x_{2}-b_{i}}{\left(x_{1}-a_{i}\right)^{2}+\left(x_{2}-b_{i}\right)^{2}}
\end{array}\right.
$$

where $a_{i}$ and $b_{i}$ are the coordinates of the radius-vector $\vec{l}_{i}$, i.e. $\vec{l}_{i}=\left(a_{i}, b_{i}\right)$.

The system (2) is a system of two rational equations (over the field of real numbers) in two variables. Equations (2) are given in Cartesian coordinates on the plane. In terms of algebraic geometry, the image of a source in N-point gravitational lens can be defined as follows:

Definition. An image of a point source in N-point gravitational lens will be called the real solution of system (2) without regard to multiplicity. The set of images is the set of different real solutions of this system.

Let us investigate the set of real solutions of system (2). We transform the equations of the system to polynomial form:

$$
\left\{\begin{array}{l}
F_{1}\left(x_{1}, x_{2}, y_{1}\right)=0 \\
F_{2}\left(x_{1}, x_{2}, y_{2}\right)=0
\end{array}\right.
$$

The equations of system (3) will be studied over the field of complex numbers. 
The system (3) is defined in. System (3) is not equivalent to system (2), but it follows from it.

We can compute the set of solutions of system (2) from the set of real solutions of system (3) if we remove solutions from it in which system (2) is not defined. These solutions are the coordinates of the point masses.

We directly check out that the points with coordinates $\left(a_{i}, b_{i}\right), i=1, \ldots, N$, are solution of system (3), but system (2) is not defined in these points.

Let $f_{1}$ and $f_{2}$ be the left-hand sides of the first and second equations of system $(2), M\left(f_{1}, f_{2}\right)$ be the solution set of system $(2), V\left(F_{1}, F_{2}\right)$ be the solution set of system (3), and $\operatorname{ReV}\left(F_{1}, F_{2}\right) \subset V\left(F_{1}, F_{2}\right)$ the subset of its real solutions, then relation:

$$
M\left(f_{1}, f_{2}\right)=\operatorname{ReV}\left(F_{1}, F_{2}\right) /\left\{\cup\left(a_{i}, b_{i}\right)\right\} .
$$

From the theorem on the structure of the set of solutions of a system of polynomial equations, see (Arzhancev, 2003) it follows that the set $V\left(F_{1}, F_{2}\right)$ can be represented in the form

$$
V\left(F_{1}, F_{2}\right)=\left(V^{0}\left(F_{1}, F_{2}\right)\right) \cup\left(V^{1}\left(F_{1}, F_{2}\right)\right),
$$

where $V^{1}\left(F_{1}, F_{2}\right)$ is the set of solutions depending on single parameter, and $V^{0}\left(F_{1}, F_{2}\right)$ is the discrete set of solutions of system (3). The dimension $\operatorname{dim} V^{1}\left(F_{1}, F_{2}\right)=1, \operatorname{dim} V^{0}\left(F_{1}, F_{2}\right)=0$.

There are several theorems that answer the question: is the set empty, see for example (Walker, 1950; Kalinina, 2002)? In (Bronza \& Kotvytskiy, 2017), we present an algorithm that allows us to describe this set analytically, if it is not empty. If the set $V^{1}\left(F_{1}, F_{2}\right)$ is not empty, then the equations of system (3) have a common component. The equation of the general component can be obtained from its analytical description.

The set $M\left(f_{1}, f_{2}\right)$ can be represented in the form: $M\left(f_{1}, f_{2}\right)=\left(M^{0}\left(f_{1}, f_{2}\right)\right) \cup\left(M^{1}\left(f_{1}, f_{2}\right)\right)$, where $M^{0}\left(f_{1}, f_{2}\right)=\operatorname{ReV}^{0}\left(F_{1}, F_{2}\right) /\left\{\cup\left(a_{i}, b_{i}\right)\right\}$ and $M^{1}\left(f_{1}, f_{2}\right)=\operatorname{ReV}^{1}\left(F_{1}, F_{2}\right) /\left\{\cup\left(a_{i}, b_{i}\right)\right\}$.

It is known that the set $M^{1}\left(f_{1}, f_{2}\right)$, for a point source in single-point lens is not empty, see for example (Bliokh et al., 1989; Zakharov, 1997; Schneider, 1999), coincides with $V^{1}\left(F_{1}, F_{2}\right)$, see (Bronza \& Kotvyskiy, 2017) and is Einstein ring. But for a point source in symmetric 2-point lens, we proved (Bronza \& Kotvyskiy, 2017) that the set $M^{1}\left(f_{1}, f_{2}\right)$ is empty and put forward hypothesis: for $\mathrm{N}$-point lens this set is empty for $N>1$.

To study the set of solutions $V^{0}\left(F_{1}, F_{2}\right)$ of system (3) we use the Bezout theorem, see for example (Walker, 1950), (Kalinina, 2002; Arzhancev, 2003; Reid, 1988).

Theorem 1. The number of intersection points of plane curves $\Phi_{1}$ and $\Phi_{2}$ is equal to $n \cdot m$, where $m=\operatorname{deg} \Phi_{1}$ and $n=\Phi_{2}$, if the curves: not have common components, are defined over an algebraically closed field, are considered on the projective plane, points of intersection are counted taking into account the multiplicity.

The Bezout theorem, in our case, is equivalent to the following

Theorem 2. The system of polynomial equations

$$
\left\{\begin{array}{l}
\Phi_{1}\left(X_{0}: X_{1}: X_{2}\right)=0 \\
\Phi_{2}\left(X_{0}: X_{1}: X_{2}\right)=0
\end{array}\right.
$$

has in the projective plane $C P^{2}$, counting multiplicity, exactly $m \cdot n$ solutions, where, $m=\operatorname{deg} \Phi_{1}$ and $n=\Phi_{2}$, if $\operatorname{gcd}\left(\Phi_{1}, \Phi_{2}\right)$ belongs to the coefficient field $C$.

In the equations of system (3) we pass to homogeneous coordinates, which are projective. Let:

$$
\left\{\begin{array}{l}
x_{1}=\frac{X_{1}}{X_{0}} \\
x_{2}=\frac{X_{2}}{X_{0}}
\end{array}\right.
$$

The system (7) defines surjective mapping, $\Im: C^{2} \rightarrow$ $C P^{2}$, which defines the left-hand sides of the equations of system (6):

$$
\left\{\begin{array}{l}
\Phi_{1}\left(X_{0}: X_{1}: X_{2}\right)=X_{0}^{2 N+1} F_{1}\left(\frac{X_{1}}{X_{0}}, \frac{X_{2}}{X_{0}}, y_{1}\right) \\
\Phi_{2}\left(X_{0}: X_{1}: X_{2}\right)=X_{0}^{2 N+1} F_{2}\left(\frac{X_{1}}{X_{0}}, \frac{X_{2}}{X_{0}}, y_{2}\right)
\end{array},\right.
$$

The functions $\Phi_{1}=\Phi_{1}\left(X_{0}: X_{1}: X_{2}\right)$ and $\Phi_{2}=$ $\Phi_{2}\left(X_{0}: X_{1}: X_{2}\right)$ are homogeneous functions of degree $2 N+1$. The triple of complex numbers $\left(X_{0}: X_{1}: X_{2}\right)$ is the coordinates of the point and specifies point $p \in C P^{2}$. The triple $\left(\lambda X_{0}: \lambda X_{1}: \lambda X_{2}\right)$ specifies the

same point if $\lambda \neq 0$. If, at least one of the coordinates of the point $p$ is equal to zero, say that this point is irregular. Otherwise, the point is called regular.

The set of points $C P^{2}$ one of the coordinates, which is equal to the number $h \neq 0$, is called affine map on $C P^{2}$ and denoted by $A^{2}(h)$. The complement of $C P^{2} \backslash A^{2}(h)$ consists of an one-dimensional complex projective subspace, which is called infinitely distant line of the affine map $A^{2}(h)$, see for example (Arzhancev, 2003; Reid, 1988). The infinitely distant line of any affine map $A^{2}(h)$ is evidently irregular.

For example, if we set Theorem 3 . In a situation of general position (the Jacobian of the system of lens equations is not equal to zero), the number of point images in N-point gravitational lens has parity opposite to the parity of the number N., then the set of points $C P^{2}$ with coordinates $\left(1: X_{1}: X_{2}\right)$ will be affine map of $A^{2}(1)$, and the infinity of the straight line of this map will be given by equation $X_{0}=0$.

We have

Theorem 3. In a situation of general position (the Jacobian of the system of lens equations is not equal to zero), the number of point images in N-point gravitational lens has parity opposite to the parity of the number $N$.

In the proof of Theorem 2 we use the following 
Lemma. The number of irregular solutions of system (6), on line $X_{0}=0$, is $2 N$.

Proof. Using (8), we reduce the system (6) to the form:

$$
\left\{\begin{array}{c}
\left(X_{1}-X_{0} y_{1}\right) \prod_{i=1}^{N}\left[\Gamma_{i}\right]-X_{0}^{2} \sum_{j=1}^{N} m_{j}\left(X_{1}-X_{0} a_{j}\right) \times \\
\times \prod_{i=1, i \neq j}^{N}\left[\Gamma_{i}\right]=0 \\
\left(X_{2}-X_{0} y_{2}\right) \prod_{i=1}^{N}\left[\Gamma_{i}\right]-X_{0}^{2} \sum_{j=1}^{N} m_{j}\left(X_{2}-X_{0} b_{j}\right) \times \\
\times \prod_{i=1, i \neq j}^{N}\left[\Gamma_{i}\right]=0
\end{array}\right.
$$

where $\Gamma_{i}=\left(X_{1}-X_{0} a_{i}\right)^{2}+\left(X_{2}-X_{0} b_{i}\right)^{2}$.

Let $X_{0}=0$. We have:

$$
\left\{\begin{array} { c } 
{ X _ { 1 } \prod _ { i = 1 } ^ { N } ( X _ { 1 } ^ { 2 } + X _ { 2 } ^ { 2 } ) = 0 } \\
{ X _ { 2 } \prod _ { i = 1 } ^ { N } ( X _ { 1 } ^ { 2 } + X _ { 2 } ^ { 2 } ) = 0 }
\end{array} \Rightarrow \left\{\begin{array}{l}
X_{1}\left(X_{1}^{2}+X_{2}^{2}\right)^{N}=0 \\
X_{2}\left(X_{1}^{2}+X_{2}^{2}\right)^{N}=0
\end{array}\right.\right.
$$$$
\Rightarrow\left(X_{1}^{2}+X_{2}^{2}\right)=0 \Rightarrow X_{1}= \pm i X_{2} \Rightarrow\left\{\begin{array}{c}
X_{1}=a \\
X_{2}= \pm a
\end{array}\right.
$$

Finally we have two $\mathrm{N}$-fold solutions: $P_{1}=$ $(0: a: i a)$ and $P_{2}=(0: a:-i a)$.

Proof of Theorem 2. For the degrees of the polynomials of systems (3) and (6) we have: $\operatorname{deg} F_{1}=$ $\operatorname{deg} F_{2}=\operatorname{deg} \Phi_{1}=\operatorname{deg} \Phi_{2}=2 N+1$. By Bezout's theorem, the system of equations $(6)$ has $(2 N+1)^{2}$ solutions, which include an even number $2 q$ of complex conjugate solutions and $P=2 N$ irregular solutions. Therefore, the number of real solutions of system (6).

$$
\begin{aligned}
& \operatorname{card}\left(\operatorname{ReV}^{0}\left(F_{1}, F_{2}\right)\right)=(2 N+1)^{2}-2 q-P= \\
& \quad=(2 N+1)^{2}-2 q-2 N=4 N^{2}+2 N+1-2 q .
\end{aligned}
$$

From the fact that the restriction of the inverse mapping $\Im^{-1}: C P^{2} \rightarrow C^{2}$ to the affine map $A^{2}(1)$ is a bijection that is given by the equations: $X_{0}=1, X_{1}=$ $x_{1}, X_{2}=x_{2}$, we have:

$$
\begin{gathered}
\operatorname{card}\left(M^{0}\left(f_{1}, f_{2}\right)\right)=\operatorname{card}\left(\operatorname{ReV}^{0}\left(F_{1}, F_{2}\right)\right)-N= \\
=4 N^{2}+N+1-2 q .
\end{gathered}
$$

In a situation of general position, the point source is not on the caustic, therefore, all elements of the set $\operatorname{ReV}\left(f_{1}, f_{2}\right)$ are different. In this case, each point of the set $\operatorname{ReV}\left(f_{1}, f_{2}\right)$ is, by definition, an image. It follows from (13) that the parity of the number of images is opposite to the parity of the number $N$. The theorem is proved.

Theorem 3 does not contradict the theorem on the oddness of the number of images in transparent lenses (Bliokh, 1989).

In the special case, for 1-point lens the number of images is: $4 N^{2}+N+1-2 q=6-2 q$ - even number; for 2-point lens: $4 N^{2}+N+1-2 q=19-2 q$ - odd number, and so on.

\section{Conclusions}

In this article, we applied methods of algebraic geometry to determine number of images in gravity lens. Proved that the parity of the number of images is opposite to the parity of the number.

\section{References}

Arzhancev I.V.: 2003, Basisy Grebnera i systemy algebraicheskikh uravneniy [in Russian]. Letnyaya shkola, Sovremennaya matematika. Moskow, P. 68.

Bliokh P.V. \& Minakov A.A.: 1989, Gravitational Lenses [in Russian], Naukova Dumka, Kiev.

Bronza S.D.: 2016, Zbirnik naukovih prats, Kharkiv, UkrURT, 68 [in Russian].

Bronza S.D., Kotvytskiy A.T.: 2017, The Journal of Kharkiv National University, ser. Physics, 26(1120), 6 .

Kalinina E.A., Uteshev A.Yu.: 2002, Teoria isklyucheniya, SPBU, Saint Petersburg, 72.

Kotvytskiy A.T., Bronza S.D.: 2016, Odessa Astron. Publ., 29, 31.

Kotvytskiy A.T., Bronza S.D., Vovk S.R.: 2016, The Journal of Kharkiv National University, ser. Physics, 24(1119), 55.

Kotvytskiy A.T., Bronza S.D., Nerushenko K.Yu., Shablenko V.Yu.: 2017, Zbirnik naukhovih prats VI Mizhregionalnoyi naukovo-praktichnoyi konferencii "Astronomiya i syogodennya", Vinnitsya, [in Ukrainian], 198.

Lang S.: Algebra. Columbia University. New York, 1965.

Reid Milies A.: 1988, Undergraduate Algebraic Geometry, 151.

Schneider P., Ehlers J., Falco E.E.: 1999, Gravitational lenses, 560.

Van Der Waerden B.L.: 1971, Algebra I, II, P. 456.

Walker R.J.: 1950, Algebraic curves, P. 236.

Zakharov A.F.: 1997, Gravitacionnye linzy $i$ mikrolinzy, 328. 